\title{
UNA NUEVA ESPECIE DEL GÉNERO CHOMELIA (RUBIACEAE, GUETTARDEAE) EN MÉXICO
}

\author{
C. H. Ramos Álvarez ${ }^{1}$ y A. Borhidi ${ }^{2}$ \\ ${ }^{1}$ Av. IMAN 580, Helios 101, México, D. F., C.P. 4510, México \\ E-mail: clara.hilda.ramos@gmail.com \\ ${ }^{2}$ Departamento de Fitotaxonomía y Geobotánica, Instituto de Biología \\ Universidad de Pécs, H-7624 Pécs, Ifjúság útja 6, Hungría. E-mail: borhidi@gamma.ttk.pte.hu
}

(Received 11 November, 2014; Accepted 15 December, 2014)

\begin{abstract}
A new species of Chomelia is described; at this time it has been collected only at the type locality. It is similar to the Mexican-Mesoamerican species Chomelia longituba (Borhidi) Borhidi, but can be easily distinguished by the position of the inflorescence, by the form of the stigma lobes and the size of the fruit.
\end{abstract}

Key words: Chomelia, Mexico, Rubiaceae, tropical rain forest, Veracruz

\section{Chomelia anisophylla Ramos et Borhidi, spec. nova, hoc loco}

(Fig. 1)

Tipo: México, Edo. Veracruz, Mpio. Coatzacoalcos, Parque ecológico Jaguaroundi, $400 \mathrm{~m}$ al $\mathrm{E}$ de los quemadores de la refinería "La Cangrejera" en el área de inventario forestal, al borde de una zona inundable. Coord.: $18^{\circ} 06^{\prime} 5.2^{\prime \prime} \mathrm{N}, 94^{\circ} 20^{\prime} 24^{\prime \prime} \mathrm{W}$, alt. $47 \mathrm{~m}$ snm. Col.: Clara H. Ramos (2769) con E. Martínez Salas y Lucía Gpe. Rivera Gómez, 14.03.2008. Holotipo: MEXU, isotipos: $\mathrm{BP}, \mathrm{MO}$.

Paratipos: Misma localidad: C. H. Ramos y E. Martínez S. (2310), 04.04.2003. Paratipo: MEXU, isoparatipos: BP, MO.

Frutex 3-6 m alta, frons rotundus, valde ramosus, cortice cinereo claro, rami cylindracei, longitudinaliter striati, lenticellis nigris punctiformibus obsita inferne ad truncum principalem spinis fortibus 1-5 cm longis suffulta, ramuli laterales breves, 2-4 cm longi, 4-angulati, canaliculati, dense albo-hirsuti, internodiis brevibus foliis apice confertis. Folia opposita in paribus longitudine petiolorum atque forma et magnitudine valde inaequalia; minora ovata vel suborbicularia petiolis tenuis 3-7 mm longis, albo-pilosis, lamina acuminata et apice acuta, basi obtusa vel rotundata 
vel leviter cordata, 1-3.5 cm longa et $0.7-1.8 \mathrm{~cm}$ lata, majora oblongo-elliptica vel lanceolata, 2-3 cm longe petiolata, petiolis albo-hirsutis suffulta, lamina inaequilata, apice obtusa vel brevissime apiculata, basi longe attenuata et saepe obliqua, 6-12 cm longa et 2-4.5 cm lata, utrinque glabra, ad nervos adpresse strigosa et subtus saepe domatiata, membranacea vel chartacea, decidua; nervi secundarii utroque latere 5-8, quaternarii dense lineolati. Lineatio subtus in foliis minoribus, in foliis majoribus utrinque conspicua. Stipulae brunneae, deltoideae vel late triangulares, cuspidatae, subulatae et aristatae, 2-4 mm longae et 2-3 $\mathrm{mm}$ latae, pars interpetiolaris basi in vaginam $1 \mathrm{~mm}$ longam connata, basi et supra aristam ferrugineo-strigosae. Vagina saepe disrupta et decidua anillum 1-1.5 mm altum e colleteribus albo-strigosis formatum revelans. Flores axillares solitarii, plerumque ad ramos laterales breves sessiles, subtenuti ab involucro tubulare angusto 0.5-1.5 $\mathrm{mm}$ longo extus hirsuto intus glabro e bracteis duabus connato apice 1-2 $\mathrm{mm}$ longe 2-aristato. Hypanthium oblongo-ellipticum, 2-3 mm longum, dense strigoso-villosum; tubus calycis 1-1.5 mm longus, lobi 4, lineares, acuti, 2.5-3 mm longi, extus hirsuti intus papillosi. Corolla alba, tubus 1-2 cm longus, tenuis et flexuosus, basi 0.6-1 mm, fauce 1.5-2 mm latus, extus pilis strigosis albis sursum directis dense copertus, intus glaber; lobi 4, linear-lanceolati et acuti, 5-7 mm longi et basi 1.5-2 mm lati, extus lineam centralem strigosi, intus glabri. Stamina 4, fauce inserta, filamentis filiformibus et brevibus, 0.4-1 $\mathrm{mm}$ longis, antherae dorsifixae, introrsae, sagittatae et caudatae, 3-4 $\mathrm{mm}$ longae, appendicibus basalibus 1-1.5 $\mathrm{mm}$ longis et $0.3 \mathrm{~mm}$ latis, linearibus et acutis. Stylus 1.4-1.6 cm longus, sub medio pilosus, stigma bilobatum, rami filiformes, $1 \mathrm{~mm}$ longi. Ovarium 1-loculare, ovulo 1, pendulo. Discus tubularis, 4-lobulatus. Fructus drupaceus 1-locularis, 1-spermus, oblongo-ovatus vel oblongo-ellipticus, $1.2-1.5 \mathrm{~cm}$ longus, purpureo-violaceus, tubo et lobulis calycinis persistentibus coronatus; meocarpium liquosum, endocarpium durum, 8-costatum. Semina 1, oblongo-elliptica vel linear-elliptica, 6-8 $\mathrm{mm}$ longa et $0.6 \mathrm{~mm}$ in diametro, apice curvata, testa laevis.

Arbusto de 3 a $6 \mathrm{~m}$ de altura, copa redonda muy ramificada, corteza gris claro; ramas cilíndricas, longitudinalmente estriadas, con lenticelas negras punctiformes, la parte inferior de los troncos principales con espinas fuertes de 1 a $5 \mathrm{~cm}$ de largo, ramas 4-angulosas, canaliculadas densamente blancohirsutas, las últimas ramillas de $2 \mathrm{a} 4 \mathrm{~cm}$ de largo, con entrenudos cortos y hojas agrupadas distalmente. Hojas opuestas, membranáceas o papiráceas, muy desiguales en cada par tanto en la longitud del pecíolo como en el tamaño y forma de las láminas. Las menores de 1 a $3.5 \mathrm{~cm}$ de largo y 0.7 a $1.8 \mathrm{~cm}$ de ancho, inequiláteras, de aovadas a suborbiculares, ápice agudo, acuminado, base obtusa, redondeada a ligeramente acorazonada; pecíolos delgados, de 3 a $7 \mathrm{~mm}$ de largo, blanco-pelosos, las hojas mayores de 6 a $12 \mathrm{~cm}$ de largo y 2 a $4.5 \mathrm{~cm}$ de ancho, de oblongo elípticas a lanceoladas, ápice obtuso o brevemente apiculado largamente estrechadas hacia la base; glabras en ambas caras ex- 


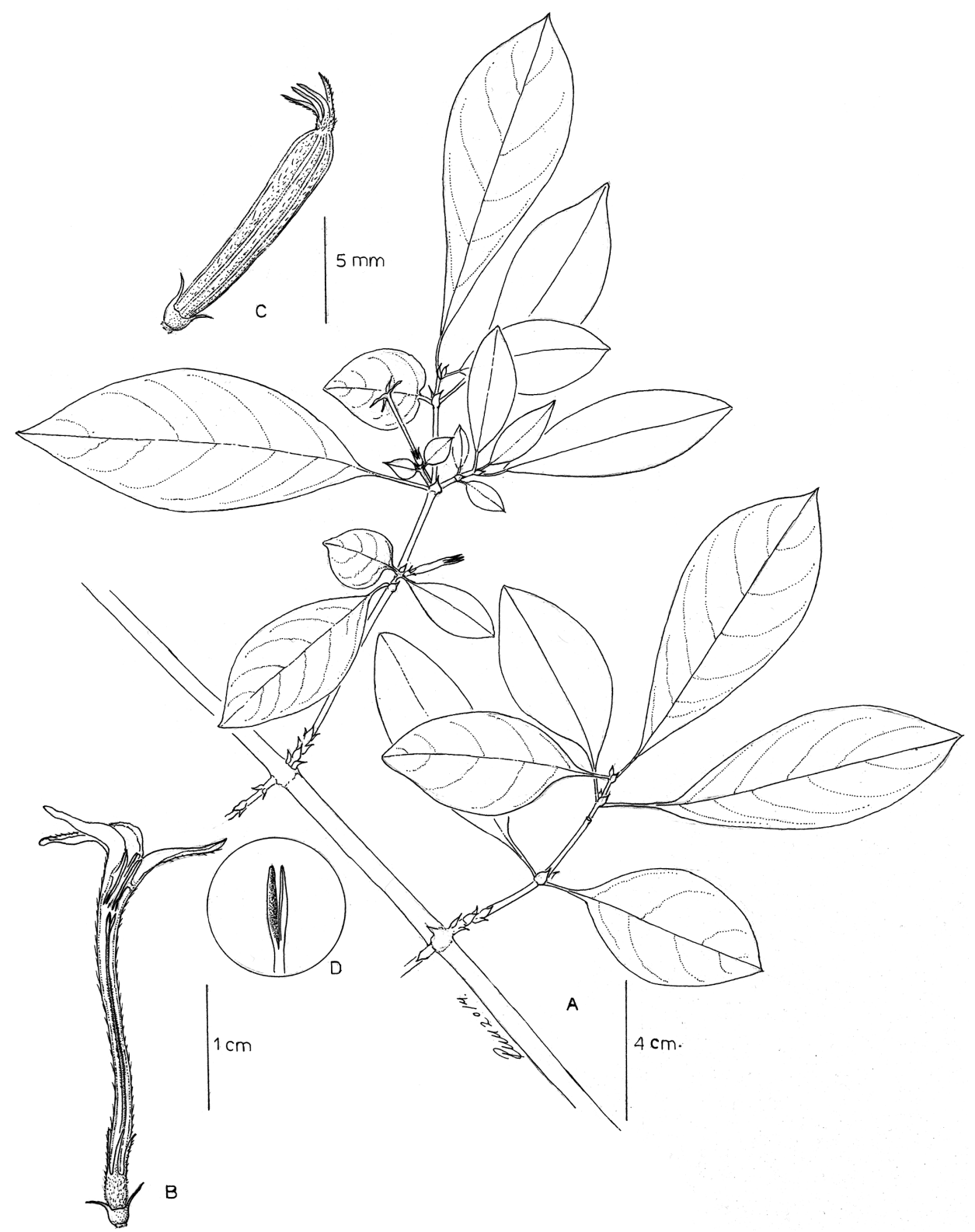

Fig. 1. Chomelia anisophylla Ramos et Borhidi, de Veracruz, México (dibujo de M. en C. Ramiro Cruz Durán). A = rama con flores (CH-2769, MEXU); B = flor (CH-2769, MEXU); $\mathrm{C}=$ fruto $(\mathrm{CH}-2310, \mathrm{MEXU}) ; \mathrm{D}=\operatorname{estigma}(\mathrm{CH}-2769 \mathrm{MEXU})$ 
cepto en las nervaduras, la principal densamente estrigosa en el envés, a menudo con domacios; nervios laterales 5 a 8 pares, los cuaternarios densamente lineolados, la lineación visible en el envés de las hojas menores y en ambas caras de las hojas mayores, membranáceas o papiráceas, caedizas; pecíolos delgados de 2 a $3 \mathrm{~cm}$ de largo. Estípulas pardas, deltadas o anchamente triangulares, de 2 a $4 \mathrm{~mm}$ de largo y 2 a $3 \mathrm{~mm}$ de ancho, aristadas, la región interpeciolar connada formando una vaina de $c a 1 \mathrm{~mm}$ de largo, ferrugíneo-estrigosa en el triángulo basal y sobre el eje de la arista. A menudo se desprende la base de la vaina, dejando libre un anillo de coléteres de 1 a $1.5 \mathrm{~mm}$ de largo, blancoestrigosos. Flores axilares, solitarias, casi siempre en las ramas laterales cortas, sésiles, subtendidas por un involucro tubular formado por dos brácteas 0.5 a $1.5 \mathrm{~mm}$ de largo, connadas, hirsutas externamente, glabras por dentro, cada bráctea con una arista de 1 a $2 \mathrm{~mm}$ de largo. Hipanto oblongo-elíptico, 2 a $3 \mathrm{~mm}$ de largo, densamente estrigoso-velloso, tubo del cáliz 1 a $1.5 \mathrm{~mm}$ de largo, lóbulos del cáliz 4, lineares, agudos, 2.5 a $3 \mathrm{~mm}$ de largo, hirsutos por fuera, papilosos por dentro. Corola blanca; tubo 1.5 a $2 \mathrm{~cm}$ de largo, estrecho y flexible, 0.6 a $1 \mathrm{~mm}$ de ancho en la base, 1.5 a $2 \mathrm{~mm}$ de ancho en la garganta, densamente cubierto por pelos estrigulosos y blancos, antrorsos, glabro por dentro; lóbulos 4, linear-lanceolados y agudos, de 5 a $7 \mathrm{~mm}$ de largo, y 1.5 a $2 \mathrm{~mm}$ de ancho en la base, estrigosos en una línea central por fuera, glabros por dentro. Estambres 4, insertos en la garganta, filamentos de 0.4 a $1 \mathrm{~mm}$ de largo, filiformes; anteras, 3 a $4 \mathrm{~mm}$ de largo, dorsifijas, introrsas, flechadas y caudadas, apéndices basales 1 a $1.5 \mathrm{~mm}$ de largo y $0.3 \mathrm{~mm}$ de ancho, lineares, agudos. Estilo de 1.4 a $1.6 \mathrm{~cm}$ de largo, peloso en la mitad inferior, estigma bilobulado, ramas de $1 \mathrm{~mm}$ de largo, filiformes. Ovario 1-locular con 1 óvulo colgante. Disco tubular, con 4 lóbulos. Fruto 1.2 a $1.5 \mathrm{~cm}$ de largo, drupáceo, 1-locular, de oblongo-obovado a oblongo-elíptico, morado, coronado por el tubo y los lóbulos persistentes del cáliz; mesocarpo jugoso, endocarpo duro, 8 -costado. Semilla 1, de 6 a $8 \mathrm{~mm}$ de largo y $0.6 \mathrm{~mm}$ de diámetro, oblongoelíptica, encorvada en el ápice, testa lisa.

Distr.: México: Veracruz, endémica.

Afín a Chomelia longituba (Borhidi) Borhidi, colectada en el estado Veracruz, Mpio. Hidalgotitlán, que difiere de nuestra planta en tener corteza casi negra, ramitas laterales cilíndricas, espinosas, ferrugíneo-estriadas, hojas iguales en cada par, flores terminales, lóbulos del cáliz y corola más largos, estigma globoso, fruto y semillas dos veces más largas.

Obs. Chomelia longituba de México fue incluida en Chomelia tenuiflora Benth. de Sudamérica en Flora Mesoamericana (2012: 56) por Taylor y Lorence, aunque las poblaciones de esta última especie no tienen continuidad en su variabilidad morfológica ni en su distribución con la especie mexicana. 
Agradecimientos - A Lucía Guadalupe Rivera, Pemex-Petroquímica por su ayuda y amable compañía en nuestras primeras visitas al Parque Jaguaroundi; al Ing. Forestal Eduardo Cardona Ureña, Director del Parque y a Hugo Cubillas y Braulio Gómez Chagala, personal del mismo por sus atenciones en nuestra reciente visita. Al Herbario Nacional MEXU, por las facilidades brindadas durante las consultas. La ilustración fue dibujada por el M. en C. Ramiro Cruz Durán, quien también aportó valiosas observaciones. Los autores dan gracias a María del Rosario García Peña por el escarneo de la imagen.

\section{LITERATURA CONSULTADA}

Borhidi, A. (2004): Especies y combinaciones nuevas de Rubiáceas en México y Mesoamerica. - Acta Bot. Hung. 46: 29-39. doi: 10.1556/ABot.46.2004.1-2.3

Borhidi, A. (2006): Rubiáceas de México. - Akadémiai Kiadó, Budapest, 512 pp.

Borhidi, A. (2012): Rubiáceas de México. Segunda y ampliada edición. - Akadémiai Kiadó, Budapest, $610 \mathrm{pp}$.

Manns, U. y Bremer, B. (2010): Towards a better understanding of intertribal relationships and stable tribal delimitations within Cinchonoideae s. s. (Rubiaceae). - Mol. Phyl. Evol. 56: 21-39. doi: 10.1016/j.ympev.2010.04.002

Taylor, C. M. y Lorence, D. H. (2012): Chomelia Jacq. - In: Davidse, G., Sousa, M., Knapp, S. y Chiang, F. (eds gen.): Flora Mesoamérica. IV(2): Rubiaceae, Cordiaceae, Boraginaceae, Lamiaceae. Universidad Nacional Autónoma de México, Missouri Botanical Garden, The Natural History Museum (London), pp. 52-56. 